\title{
Anmerkungen zum Q-Monitoring der FMH
}

\author{
Josef Babics, Markus Löliger, \\ Astrid Lyrer, Peter Minder, \\ Walter Nef, Esther Ramseyer, \\ Peter E. Schlageter, Gian-Clo \\ Serena, Peter Strohmeier, \\ Philipp Zinsser
}

\section{Einleitung}

Im Qualitätszirkel Oberwil (QZO) arbeiten zur Zeit 10 Hausärztinnen und Hausärzte. Die Sitzungen finden monatlich statt. Bei unserer Arbeit befolgen wir die SGAM/SGIM-Richtlinien: Wir bestimmen ein praxisrelevantes Thema, überprüfen unsere Praxis und suchen allfällige Lücken und Fehler zu korrigieren, um damit unsere Arbeit zu verbessern. Die freiwillige Teilnahme am Qualitätszirkel ist für uns alle ein wichtiger, jedoch längst nicht der einzige Bestandteil unserer Qualitätssicherung in der hausärztlichen Praxis.

\section{Qualitätsmonitoring der FMH}

Das Ressort Daten Demographie Qualität (DDQ) der FMH hat im Februar 2010 eine Internet-basierte Umfrage zum Thema Qualitätssicherung bei Grundversorgern und Psychiatern durchgeführt [1]. Diese Umfrage hat unter den Teilnehmern unseres Zirkels Irritationen ausgelöst.

- Wir haben uns darüber gewundert, welch breiten Raum die Fragen nach externen Qualitätsaktivitäten eingenommen haben. Die Umfrage liess annehmen, die FMH plane die Einführung von Zertifizierungen durch externe Anbieter ausserhalb der Fachgesellschaften.

- Im Gegensatz dazu fehlte im Fragebogen die Möglichkeit zu kommentieren, welche der aufgelisteten Qualitätsaktivitäten wir für relevant halten dass es in unserer täglichen Arbeit messbare und nicht messbare Qualität gibt.

Messbar ist die Qualität bei der Infrastruktur und im apparativ/technischen Bereich. So ist Qualität im Labor und beim Röntgen mit der Wartung der Geräte und externer Laborkontrolle kontrollierbar und somit «messbar». Auch bei der Infrastruktur einer Praxis sind messbare Qualitätskriterien vorhanden. Angefangen bei Trivialem, wie genügend Sitzgelegenheiten im Wartezimmer, bis zu Wichtigerem wie rollstuhlgängigem Zutritt zur Praxis. Schon hier stellt sich aber die Frage, wie wichtig das Messen von Qualität wird, wenn «Triviales» erfasst wird oder aber Wichtiges zwar erkannt, aber nicht verändert werden kann (z.B. Verkehrssituation, Anbindung an den öffentlichen Verkehr usw.).

Noch problematischer erscheint uns das Messen von Qualität, wenn klare Qualitätskriterien fehlen: Erreichbarkeit, Wartezeiten, Teilnahme an Fortbildung, persönliche Vernetzung, Art der Krankengeschichte sind zwar messbar oder sie können evaluiert werden. Aber: Sagen wir mit dem «Messen» dieser Grössen wirklich etwas aus über die Qualität unserer täglichen Arbeit?

Das möglicherweise wichtigste Instrument zur Erhaltung und Verbesserung der hausärztlichen Behandlungsqualität ist die Fortbildung. Sie wird von den Hausärzten dokumentiert und deklariert. Wir alle
Nikolic V. Q-Monitoring Start der Datenerfassung Interview mit Dr. med. Daniel Herren. Schweiz Ärztezeitung. 2010; 91(4): 112-3.

2 Nikolic V, Herren D. Die Ärzteschaft sichert Qualität und wie! Schweiz Ärztezeitung. 2010;91(25):973-6.

3 Peltenburg M et al. Qualität ein Zusammenspiel aller Kräfte im ärztlichen Umfeld. Schweiz Ärztezeitung. 2005 86(18):1055-9. Mindmap: Leitbild Ärztlicher Qualität. www.saez.ch/mindmap.pdf

4 Ressort DDQ. Grundlagenpapier der AGQ-FMH: Qualitätstransparenz. Schweiz Ärztezeitung. 2009; 90(26/27):1037-40.

Korrespondenz:

Dr. med. Astrid Lyrer-Gaugler Moderatorin QZ Oberwil Allgemeine Medizin FMH Feierabendstrasse 74 CH-4051 Basel

astrid.lyrer@bluewin.ch

\section{Vor allem Peer Review und CIRS im Qualitätszirkel sind gute und geprüfte Instrumente zur Entwicklung der Qualität}

und welche nicht. Wir konnten uns nicht zur Qualität der Umfrage selber äussern.

- Es war nicht klar ersichtlich, was die FMH mit den einmal erhobenen Daten zu tun gedenkt.

- Qualität sei eine Koproduktion, hält die FMH fest [2]. Nicht alle von der FMH für die Begleitung des Projektes ausgewählten «Partner» haben sich bisher durch Interesse oder durch Erfahrung mit Qualität in der Hausarztmedizin ausgezeichnet.

\section{Qualität in der Hausarztmedizin}

Wir haben uns deshalb entschieden, das Thema «Qualität in der Hausarztmedizin» in unserem Qualitätszirkel zu bearbeiten. Wir wollten wissen, wie wir in unserer täglichen Praxis Qualität definieren und was die Kriterien für eine gute Qualität sind. Wir haben die Literatur gesichtet und unsere eigenen Qualitätsansprüche definiert [3, 4]. Dabei stellten wir fest, bilden uns fort. Die «gemessenen» Fortbildungsstunden übersteigen bei den meisten das obligatorische Mass bei weitem.

Darüber hinaus arbeiten die meisten Hausärzte in einem Qualitätszirkel mit. Diese Form der Fortbildung hat in der Schweiz vor ca. 20 Jahren Fuss gefasst und sich als eines der wichtigsten und bewährtesten Instrumente zur Qualitätssicherung in der Hausarztmedizin etabliert. Wir wissen aus Erfahrung, dass nicht die Anzahl Stunden, sondern der Inhalt der Fortbildung und dessen Implementation in die tägliche Arbeit für die Entwicklung der Qualität entscheidend ist. Erfahrungsgemäss fliesst in Vorträgen vermitteltes Wissen in geringem Umfang in die tägliche Praxis ein. Dies steht im Gegensatz zu dem in Peer Groups erarbeitetem Konsens, der in der Praxis eher umgesetzt wird. Dass in unserem Qualitätszirkel ausserdem ein Critical Incident Reporting System (CIRS) 
Es gibt Bereiche, da ist Qualitätsmonitoring einfach. Bei der Hausarztmedizin ist dies jedoch schwieriger.

5 Ressort DDQ. Qualitätsmanagement und Zertifizierung der ärztlichen Leistungserbringung: Aufwand und Nutzen. Schweiz Ärztezeitung. 2008;89(20):838-40.

6 Zoller M, Dahinden A. Qualitätszirkel in der Schweiz: Wohin geht die Entwicklung? PrimaryCare.

2004;4(35/36):663-5.

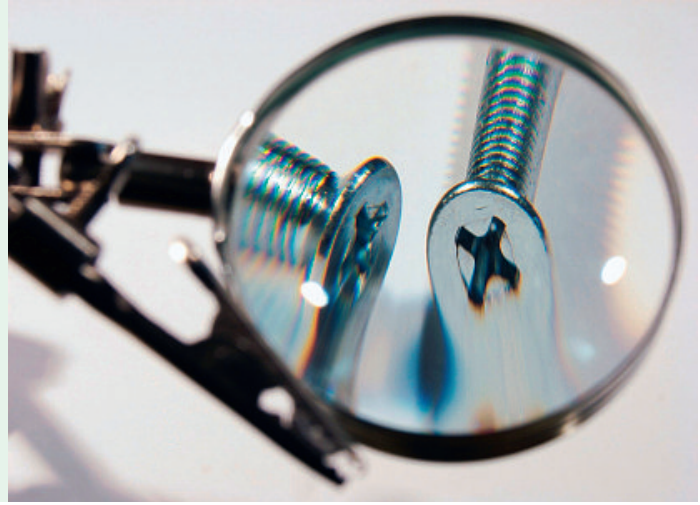

integriert ist, erachten wir als selbstverständlich. Die qualitativ grösste Herausforderung in der hausärztlichen Praxis ist die Analyse und Bearbeitung komplexer Krankheits- und Lebenssituationen, in denen sich unsere Patienten befinden.

«The Fascination of Complexity - Dealing with Individuals in a Field of Uncertainty» war denn auch das Thema des Wonca-Kongresses 2009 in Basel. Die Fähigkeit zur Bearbeitung dieser Komplexität ist nicht mit einfachen und reproduzierbaren Methoden messbar. Ebenso wenig wie die Freundlichkeit des Personals, die soziale und kommunikative Kompetenz des Arztes, die Kenntnis der ständig wechselnden «guten» Behandlungspfade, die Kenntnis der «richtigen» Personen und Institutionen (wir wollen die richtigen Türen öffnen) und das Erarbeiten eines umfassenden persönlichen Netzwerkes, in dem wir arbeiten.

\section{Fazit}

Als Schlussfolgerung stellen wir die Frage, wie Qualität transparent gemacht werden soll, wenn die wich- tigsten Qualitäts-Faktoren nicht messbar und auch nicht zu evaluieren sind.

Wir haben den Eindruck, als stünden wir mitten in einer «Qualitätsoffensive».

In ihrem Positionspapier «Qualitätsmanagement und Zertifizierung der ärztlichen Leistungserbringung: Aufwand und Nutzen» [5] schreibt das Ressort DDQ der FMH, dass dem Aufwand (nämlich Kosten, Zeit, Personalaufwand usw.), der bei Qualitätsmanagement-Systemen und Zertifizierungen anfällt, ein Nutzen gegenüberstehen müsse. Weiter hält das Ressort fest: «Unseres Wissens wurden bisher weder der Nutzen der Qualitätsmanagementsysteme noch der Zertifizierung auf die Qualität der ärztlichen Leistungserbringung systematisch untersucht und erwiesen.»

Das Qualitätsmonitoring des Ressorts DDQ in der Hausarztmedizin muss unseres Erachtens neu fokussiert werden. Die Ausrichtung und Beschränkung auf das Wesentliche ist dringend geboten, um hausärztliche Qualitätssicherung adäquat und relevant abbilden zu können.

Permanente Fortbildung, technische Qualitätskontrollen (z.B. Röntgen, externe Labor-Resultatskontrollen), ganz besonders jedoch Peer Review und CIRS im Qualitätszirkel sind gute und geprüfte Instrumente zur Entwicklung der Qualität. Das tun wir mit Überzeugung, Elan und Freude.

Die externe «Qualitätsindustrie» jedoch, eine eigentliche Art Schattenwirtschaft mit teilweise berufsfremden Akteuren, verbunden mit Bürokratie, Umtrieben und Kosten zulasten der Hausärzte, leistet unseres Erachtens keinen gesicherten Beitrag zu besserer Qualität.

Quis custodiet custodes? (Juvenal 1./2. Jh. p. Chr.: Wer wird die Wächter bewachen?)

\section{Kommentar des Ressorts DDQ \\ zum vorangegangenen Beitrag}

Die Qualität der Hausarztmedizin in der Schweiz ist hoch und viele motivierte und gutausgebildete Kolleginnen und Kollegen kümmern sich täglich mit viel Engagement um ihre Patienten. In der Welt des Qualitätsmanagements stellt man rasch fest, dass bei medizinischen Leistungen verschiedene Dimensionen der Qualitätsbeurteilung möglich sind. Am einfachsten zu beurteilen sind Strukturindikatoren, gefolgt von Prozessparametern. Beide können relativ gut abgebildet werden - wirklich schwierig wird es aber bei den beiden anderen Dimensionen Ergebnisqualität und Indikationsqualität. Wenn der Qualitätsnachweis in Hausarztpraxen auf das Mitmachen in Qualitätszirkeln und der Absolvierung der obligatorischen Fortbildung beschränkt wird, wird man der Komplexität der Qualität einer Patientenbehandlung nicht gerecht. Zudem sind technische Gerätekontrollen gesetzliche Vorschrift und haben mit dem eigentlichen Qualitätsmanagement wenig zu tun. Das Projekt Q-Monitoring, welches das Ressort DDQ der FMH zusammen mit den Qualitätsspezialisten der Hausarztmedizin, den Psychiatern und den Orthopäden durchgeführt hat, hat einzig zum Ziel, aufzuzeigen, welche Qualitätsaktivitäten in den ambulanten Praxen heute durchgeführt werden. Es ist der erste Schritt in einer Bestandesaufnahme solcher Aktivitäten, nicht mehr und nicht weniger, da gibt es keine «hidden agenda» der FMH. Weiterführend evaluieren wir die existierenden Qualitätsaktivitäten nach ihrer Wertigkeit und ihrem Aufwand-Nutzen-Verhältnis. Die FMH versteht sich hier als Drehscheibe und Vermittlerin von bereits bestehendem Wissen und will koordinierend helfen, neue Konzepte zu erarbeiten. Q-Monitoring bringt den Fachgesellschaften insofern einen Mehrwert, dass Qualitäts-Projekte sowie die Fortbildungen in Zukunft gezielter angegangen werden können, und bestehende Lücken geschlossen werden. Für die FMH ganz wichtig sind diese Umfrageergebnisse in der Umsetzung von politischen Projekten. So fliesst zum Beispiel das Wissen aus dem Projekt Q-Monitoring direkt in die Diskussion um die Konkretisierung der Qualitätsstrategie des Bundes ein und soll helfen, unsinnige Aktivitäten zu verhindern und sinnvolle zu unterstützen. So oder so, der Druck zum Qualitätsnachweis ist auch in der Hausarztpraxis massiv gestiegen, und es ist der klare Wille des Bundes, bestehende Verordnungen wie den Artikel 22a des Krankenversicherungsgesetzes (KVG), der eine Qualitätstransparenz fordert, durchzusetzen. Zu fürchten haben wir freilich nichts: Die Hausarztmedizin tut viel Gutes, wir müssen es jedoch gegen aussen dokumentieren.

Dr. med. Daniel Herren, MHA, Mitglied des Zentralvorstandes der FMH, Verantwortlicher für das Ressort DDQ 